\title{
Generalized Model of Pulse Process for Dynamic Analysis of Sylov's Fuzzy Cognitive Maps
}

\author{
R.A. Isaev ${ }^{1}$, A.G. Podvesovskii ${ }^{1}$ \\ ${ }^{I}$ Bryansk State Technical University, 50 let Oktyabrya boul. 7, 241035, Bryansk, Russia
}

\begin{abstract}
Pulse process as a means of dynamic analysis of cognitive models of semi-structured systems is studied. There is introduced and substantiated a generalized model of pulse process for Sylov's fuzzy cognitive maps, suggested its implementation for various semantic interpretations of concept interactions. The results of experimental validation of the proposed models are given.
\end{abstract}

Keywords: cognitive modeling; fuzzy cognitive map; dynamic analysis; pulse process

\section{Introduction}

One of the approaches to study semi-structured systems, widely used at the present time, is a cognitive approach. In accordance with the definition given in [1], this approach focuses on the development of formal models and methods supporting the intelligent process of solving problems because these models and methods take into account human cognitive capabilities (perception, conception, cognition, understanding, explanation) in solving management problems. Methods of structured, target and simulation modeling on the basis of cognitive approach are commonly combined by the general term "cognitive modeling". In general, cognitive modeling refers to the study of the structure of a system and the processes of its functioning and development by analyzing its cognitive model. The cognitive model of a system is based on a cognitive map, which reflects the subjective view of the researcher about it (individual or collective) as a set of semantic categories (called factors or concepts) and a set of cause-and-effect relations between them.

A cognitive model is an effective tool for exploration and estimation analysis of the situation. It does not give the opportunity to obtain accurate quantitative characteristics of the system under study, but it allows to assess the trends related to its functioning and development, and to identify significant factors influencing these processes mostly. Thanks to this we can search, generate and develop effective solutions for managing the system, as well as identify risks and develop strategies to reduce them.

Cognitive modeling starts with creating a cognitive map of the system under study on the basis of information received from experts. The next step includes directly modeling, which main objectives are forming and testing hypotheses of the system structure under study that can explain its behavior as well as developing strategies for situations in order to reach the specified targets.

The tasks solved by means of cognitive modeling can be divided into two groups:

1. The tasks of structured and target analysis:

- finding the factors which have the most significant influence on targets;

- identification of contradictions between the targets;

- identification of feedback loops.

2. The tasks of dynamic analysis (scenario modeling):

- self-development (“what if nothing is done?");

- managed development:

○ direct task ("what if ...?");

○ inverse task ("how to do ...?").

Thus, with the help of scenario modeling it is possible to predict the state of the simulated system under different management actions as well as the search for alternative management solutions to bring the system to the target state.

The most common mathematical apparatus used to represent cognitive models and being the base of the methods for their analysis is fuzzy logic. Because of this there appeared a class of cognitive models based on different types of fuzzy cognitive maps (FCM) - a very detailed overview of such models can be found in monograph [3]. One of FCM varieties, well-proven in practical problems of analyzing and modeling of ill-structured organizational, social and economic systems are Sylov's FCM firstly proposed in [7] and representing the development of signed cognitive maps [6]. For this type of FCM there was developed quite a wide range of methods of structured and target analysis based on the study of such FCM factors as consonance, dissonance and action. A detailed description of these methods can be found in the original monograph [7], and some examples of their application in the study of different organizational and social systems - in papers $[2,4]$. The problem of developing and improving dynamic analysis methods of Sylov's FCM was given far less attention. This article discusses the approach to dynamic analysis of this type FCM with the use of a generalized model of pulse process. The proposed approach is based on the notion of pulse process, originally introduced in [6] for the class of signed cognitive maps, generalizing this concept by extending it to the class of FCM, and is a development of the approach, first mentioned in [5] and is described in more detail in monograph [4] (section 3.2). 


\section{Formal definition and structure of Sylov's fuzzy cognitive map}

As it has already been mentioned, the cognitive model is based on formalization of cause-and-effect relations which occur between the factors characterizing the system under study. The result of formalization is representing the system in the form of cause-and-effect network, called a cognitive map and having the following form:

$$
G=<E, W>\text {, }
$$

where $E=\left\{e_{1}, e_{2}, \ldots, e_{K}\right\}$ is a set of factors (also called concepts), $W$ is a binary relation over set $E$, which specifies a set of cause-and-effect relations between its elements.

Concepts can specify both relative (qualitative) characteristics of the system under study, such as popularity, social tension, and absolute, measurable values - population size, cost, etc. Besides, every concept $e_{i}$ is connected with state variable $v_{i}$, which specifies the value of the corresponding index at a particular instant. State variables can possess values expressed on a certain scale, within the established limits. Value $v_{i}(t)$ of state variable at instant $t$ is called the state of concept $e_{i}$ at the given instant. Thus, the state of the simulated system at any given instant is described by the state of all the concepts included in its cognitive map.

Concepts $e_{i}$ and $e_{j}$ are considered to be connected by relation $W$ (designated as $\left(e_{i}, e_{j}\right) \in W$ or $\left.e_{i} W e_{j}\right)$ if changing the state of concept $e_{i}$ (cause) results in changing the state of concept $e_{j}$ (effect). In this case we say that concept $e_{i}$ has an influence on concept $e_{j}$. Besides, if increasing the value of the state variable of concept-cause leads to increasing the value of the state variable concept - effect, then the influence is considered positive (amplification), and if the decrease - negative (inhibition). Thus, relation $W$ can be represented as a union of two disjoint sets $W=W^{+} \quad W^{-}$, where $W^{+}$is a set of positive relations and $W^{-}$is a set of negative relations.

Fuzzy cognitive model is based on the assumption that the influence between concepts may vary in intensity, besides, this intensity may be constant or variable in time. In order to take into account this assumption, $W$ is set as a fuzzy relation, besides, the way of its setting depends on the adopted approach to formalization of cause-and-effect relations. Cognitive map with fuzzy relation $W$ is called a fuzzy cognitive map.

Sylov's fuzzy cognitive map represents FCM, characterized by the following features.

1. State variables of concepts can possess values on the interval $[0,1]$.

2. The intensity of interactions is considered constant, so relation $W$ is specified as a set of numbers $w_{i j}$, characterizing the direction and degree of intensity (weight) of influence between concepts $e_{i}$ and $e_{j}$ :

$$
w_{i j}=w\left(e_{i}, e_{j}\right) \text {, }
$$

where $w$ is a normalized index of influence intensity (characteristic function of relation $W$ ) with the following properties:

a) $-1 \leq w_{i j} \leq 1$;

b) $w_{i j}=0$, if $e_{j}$ does not depend on $e_{i}$ (no influence);

c) $\quad w_{i j}=1$ if the positive influence of $e_{i}$ on $e_{j}$ is maximum, i.e. when any changes in the system related to concept $e_{j}$ is univocally determined by the actions associated with concept $e_{i}$;

d) $w_{i j}=-1$ if negative influence is maximum, i.e. when any changes related to concept $e_{j}$ are clearly constrained by the actions associated with concept $e_{i}$;

e) $w_{i j}$ possesses the value from the interval $(-1,1)$, when there is an intermediate degree of positive or negative influence.

It is easy to notice that FCM of this structure can be graphically represented as a weighted directed graph, which points correspond to the elements of set $E$ (concepts) and arcs - to nonzero elements of relation $W$ (cause-and-effect relations). Each arc has a weight which is specified by the appropriate value $w_{i j}$. In this case, relation $W$ can be represented as a matrix of dimension $n \times n$ (where $n$ is a number of concepts in the system), which can be considered as the adjacency matrix of the graph and is called a cognitive matrix. In addition, each point of the graph also has a weight which corresponds to the concept state and can change over time.

\section{Pulse process as a means of dynamic analysis of cognitive maps}

The basis of dynamic analysis of cognitive maps is modeling of dynamics of concept states over time. Besides, concept state may change, firstly, due to changes of state of other concepts influencing this one, and, secondly, due to external influences. External influence on the concept is understood as change of its state relative to the current one under the impact of external factors, i.e. irrelatively the concepts included in the cognitive map. At the same time external influence can be targeted, i.e. it comes from the subject carrying out management of the system, and untargeted, i.e. due to external factors to the system which are beyond control. Accordingly, in the first case we will talk about control action, and in the second case - about perturbation action (or perturbations).

To describe the dynamics of concept states we will use pulse processes. The basis of this approach is the assumption that changes of all concept states occur at discrete moment of time. State change of concept of $e_{i}$ at instant $t$ will be called pulse and denote as $p_{i}(t)$. Thus,

$$
p_{i}(t)=v_{i}(t)-v_{i}(t-1)
$$

Additionally, it is assumed that influence is transmitted by one step: changing the state of the concept-cause at instant $t$ result in changing the state of the concept-effect at instant $t+1$.

Let us first give the model of pulse process for signed cognitive maps, i.e. maps which take into account only the directions of influence and do not take into account their intensity. For these maps values $w_{i j}$ can only take values -1 , 0 or 1 , and respectively, the graph arcs are marked by signs "+" and "_". The model of pulse process was proposed in [6]: 


$$
\begin{aligned}
& \text { Data Science / R.A. Isaev, A.G. Podvesovskii } \\
& \qquad p_{i}(t+1)=\sum_{j=1}^{K} \operatorname{sgn}\left(w_{j i}\right) p_{j}(t),
\end{aligned}
$$

accordingly

$$
v_{i}(t+1)=v_{i}(t)+\sum_{j=1}^{K} \operatorname{sgn}\left(w_{j i}\right) p_{j}(t) .
$$

Thus, changing the state (pulse) of each concept in the current step is determined by the pulses of all concepts influencing it and by the ratio of influence signs. Besides, transfer of positive influence is neutralized by simultaneous transfer of negative influence, and vice versa.

In $[4,5]$, a modified model of pulse process for Sylov's FCM was proposed. The model takes into account the transfer of influences between concepts and external influences:

$$
v_{i}(t+1)=\min \left(v_{i}(t)+u_{i}(t+1)+q_{i}(t+1)+\sum_{j=1}^{K} w_{j i} p_{j}(t), 1\right),
$$

where $u_{i}(t+1)$ is control action on concept $e_{i}$ at instant $t+1 ; q_{i}(t+1)-$ is disturbance $e_{i}$ at instant $t+1$.

\section{Generalized model of pulse process}

In the framework of model (1) it is assumed that the state change of concept $e_{j}$ is equal to the difference between its state at the current step and the previous step:

$$
p_{j}(t)=v_{j}(t)-v_{j}(t-1)
$$

Thus, in dynamic modeling in order to determine the state of dependent concepts we take into account absolute change of states of influencing concepts. This approach is acceptable, but at the same time, it is not the only possible one. In this regard, it is advisable to consider other, alternative approaches to interpret the interaction of concepts and propose alternative models of pulse process on their basis.

However, it is necessary to define a number of requirements to models of pulse process, which must be met by all proposed models in the future, regardless of the assumptions which they are based on.

Firstly, the model of pulse process should unambiguously determine the state of arbitrary concept $e_{i}$ at instant $(t+1)$, using for this purpose the following available information:

- the state of the same concept $e_{i}$ at instant $t$;

- the state of concepts $e_{j}, \ldots, e_{k}$, influencing concept $e_{i}$, at instant $t$;

- the state of these concepts influencing $e_{i}$, at instant $(t-1)$;

- connection weights (influence intensity) $w_{j i}, \ldots, w_{k i}$ between all dependent concepts and $e_{i}$;

- control and disturbance influences on $e_{i}$ at instant $(t+1)$, if there are any.

Or, more formally:

$$
v_{i}(t+1)=f\left(v_{i}(t), v_{j}(t), \ldots, v_{k}(t), v_{j}(t-1), \ldots, v_{k}(t-1), w_{j i}, \ldots, w_{k i}, u_{i}(t+1), q_{i}(t+1)\right) .
$$

Secondly, the following conditions should be met:

- the values of state variables of concepts should belong to the interval $[0,1]$, that is $v_{i}(t+1) \in[0,1]$;

- if influence intensity between concepts $e_{j}$ and $e_{i}$ is equal to 0 , then changing $e_{j}$ state should not cause changing $e_{i}$ state;

- if the state of influencing concepts at the previous step did not change $\left(v_{j}(t)=v_{j}(t-1)\right.$ for all $\left.j\right)$, and there is no control and disturbance influence, then the state of the dependent concept at the current step should not change: $v_{i}(t+1)=v_{i}(t)$;

- when increasing (decreasing) the state of influencing concept and the positive relation, the state of dependent concept should not decrease (not increase): $v_{i}(t+1) \geq v_{i}(t)$ if $w_{j i}>0$ and $v_{j}(t)>v_{j}(t-1) ; v_{i}(t+1) \leq v_{i}(t)$ if $w_{j i}>0$ and $v_{j}(t)<v_{j}(t-1)$

- when increasing (decreasing) the state of influencing concept and the negative relation, the state of dependent concept should not increase (not decrease): $v_{i}(t+1) \leq v_{i}(t)$ if $w_{j i}<0$ and $v_{j}(t)>v_{j}(t-1) ; v_{i}(t+1) \geq v_{i}(t)$ if $w_{j i}<0$ and $v_{j}(t)<v_{j}(t-1)$

- more significant change of the influencing concept with other things being equal should result in more significant change of the dependent concept: $p_{i}^{1}(t+1) \geq p_{i}^{2}(t+1)$, if $p_{j}^{1}(t) \geq p_{j}^{2}(t)$;

- higher intensity of the influence with other things being equal should result in more significant change of the dependent concept: $p_{i}^{1}(t+1) \geq p_{i}^{2}(t+1)$, if $w_{j i}^{1} \geq w_{j i}^{2}$.

The expression (2) together with the above mentioned conditions we call a generalized model of pulse process. This model, on the one hand, comprises model (1) as a possible particular case, and on the other hand, it creates the base for building other implementations of pulse process model. 


\section{Implementation of the generalized model of pulse process}

Let us consider the alternative implementations of the described generalized model of pulse process, involving different interpretations of concept interaction.

\subsection{Model of impulse process, based on relative changes of concept states}

We assume that concept influence on the system is determined not by changes of its condition in general, but how significant is this change relative to the previous state of this concept. In other words, we consider the relative state changes of the concepts, not absolute one.

With this purpose we will consider pulse $p_{i}(t)$ as a relative state change of concept $e_{i}$ at instant $t$ :

$$
p_{i}(t)=\frac{v_{i}(t)-v_{i}(t-1)}{v_{i}(t-1)} .
$$

Thus, the value of pulse $p_{i}(t)$ shows what fraction of its state concept $e_{i}$ changes at instant $(t-1)$.

Now, let us define the way of transferring influence between directly related concepts. Let there is a relation between concepts $e_{j}$ and $e_{i}$, which strength is equal to $w_{j i}$. At the beginning, knowing $p_{j}(t)$ - relative change of state $e_{j}$ at instant $t$, let us define the relative change of state $e_{j}$ at instant $(t+1)$.

It is necessary to consider the conditions of the generalized model, and the following additional conditions:

- if $p_{j}(t)=0$ or $w_{j i}=0$, then $p_{i}(t+1)=0$;

- $\quad$ if $w_{j i}=1$, then $p_{i}(t+1)=p_{j}(t)$.

The following product satisfies these conditions:

$$
p_{i}(t+1)=w_{j i} p_{j}(t)
$$

Finally, let us define the state of concept $e_{j}$ at instant $(t+1)$. Note that

So,

$$
p_{i}(t+1)=\frac{v_{i}(t+1)-v_{i}(t)}{v_{i}(t)} \text {. }
$$

$$
v_{i}(t+1)=v_{i}(t)+v_{i}(t) w_{j i} p_{j}(t)
$$

The resulting model is easily generalized in the case of multiple influencing concepts:

$$
v_{i}(t+1)=v_{i}(t)+v_{i}(t) \sum_{j=1}^{K} w_{j i} p_{j}(t) .
$$

As one of the conditions of the generalized model is that the concept states are within the interval $[0,1]$, then we should add the following constraints to the model:

$$
v_{i}(t+1)=\max \left(\min \left(v_{i}(t)+v_{i}(t) \sum_{j=1}^{K} w_{j i} p_{j}(t), 1\right), 0\right) .
$$

Besides, control and disturbance influences on $e_{i}$ should also be defined in terms of relative changes. For example, control influence $u_{i}(t+1)=0,1$ means "to increase the value of a state variable of $i$-concept by $10 \%$ of its current value".

Thus, we obtain the final version of the model:

$$
v_{i}(t+1)=\max \left(\min \left(v_{i}(t)+v_{i}(t) u_{i}(t+1)+v_{i}(t) q_{i}(t+1)+v_{i}(t) \sum_{j=1}^{K} w_{j i} p_{j}(t), 1\right), 0\right) .
$$

\subsection{Multiplicative model of pulse process}

Let us consider another model which also takes into account relative changes of concept states but implies slightly different interpretation of these changes. This model is not equivalent to that one described above, but they both come from similar preconditions.

In this case, relative state change of concept $e_{j}$ shows how much this concept has changed at instant $t$ compared with its condition at instant $(t-1)$ :

$$
p_{i}(t)=\frac{v_{i}(t)}{v_{i}(t-1)} .
$$

Let us define the way of transferring influence between directly related concepts In addition to the terms of the generalized model, in this case, the following conditions should be taken into account:

- $\quad$ if $w_{j i}=1$, then $p_{i}(t+1)=p_{j}(t)$;

- $\quad$ if $w_{j i}=0$ or $p_{j}(t)=1$, then $p_{i}(t+1)=1$; 
- if $w_{j i}=-1$, then $p_{i}(t+1)=\frac{1}{p_{j}(t)}$.

Operation of exponentiation satisfies these conditions:

$$
p_{i}(t+1)=\left(p_{j}(t)\right)^{w_{j i}} .
$$

Now it is easy to determine the state of concept $e_{i}$ at instant $(t+1)$ :

$$
v_{i}(t+1)=v_{i}(t)\left(p_{j}(t)\right)^{w_{j i}} .
$$

Generalization of the model in the case of multiple influencing concepts will be the following:

$$
v_{i}(t+1)=v_{i}(t) \prod_{j=1}^{K}\left(p_{j}(t)\right)^{w_{j i}} .
$$

This model does not use negative values (excluding connection weights used as indexes), thus, fulfillment of condition $v_{i}(t+1) \geq 0$ is guaranteed. To fulfill the other condition of the generalized model, namely $v_{i}(t+1) \leq 1$, we add the constraint:

$$
v_{i}(t+1)=\min \left(v_{i}(t) \prod_{j=1}^{K}\left(p_{j}(t)\right)^{w_{j i}}, 1\right) .
$$

Control influence and disturbance in this model should be specified on the basis of interpretation "a concept state has changed $n$ times". For example, control influence $u_{i}(t+1)=2$ means "to increase the concept state 2 times in comparison with the current state".

So, here is the final version of the model:

$$
v_{i}(t+1)=\min \left(v_{i}(t) u_{i}(t+1) q_{i}(t+1) \prod_{j=1}^{K}\left(p_{j}(t)\right)^{w_{j i}}, 1\right) .
$$

\section{Experimental validation of the model of pulse process under study}

For the purpose of experimental validation and comparison of the examined models, let us perform dynamic analysis of cognitive maps using each of them with the same initial data.

Fig. 1 shows a fragment of the cognitive map used for the experiment. Connection weights have the following values: $w_{12}=0,9 ; w_{23}=-0,8 ; w_{31}=0,7$. Initial concept states are specified as the following: $v_{1}(1)=0,2 ; v_{2}(1)=0,3 ; v_{3}(1)=0,8$.

Let there is control influence on concept 1 , which results in its transfer into state $v_{1}(2)=0,6$. Under the influence of the initial pulse, concept states begin to change in accordance with the rules defined by each model of pulse process.

Fig. 2-4 give schedules of changes of concept states during operation of three models of pulse process. The horizontal axis shows modeling steps, the vertical axis shows the state of the appropriate concept. Schedules have the following signs:

- "Model 1" - the results obtained using the additive model (1);

- "Model 2" - the results obtained using the additive model (3) based on the relative state changes of concepts;

- "Model 3" - the results obtained using the multiplicative model (4) based on the relative state changes of concepts.

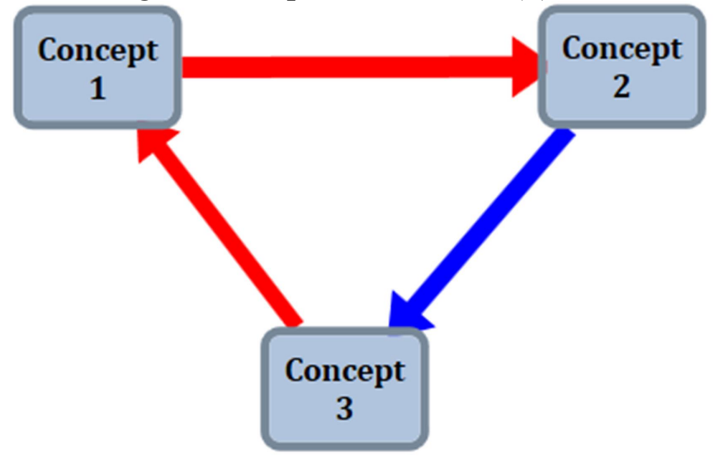

Fig. 1. Fragment of a fuzzy cognitive map used for the experiment.

Of the greatest interest for the interpretation is the transfer of influence between directly related concepts, differently occurring in the framework of different models, which result in different results in the end. Thus, in models 2 and 3 , implying relative change of concept states, the state of the second concept on the 3rd modeling step increased more than in model 1. Similarly, relative changes result in more significant decrease in the state of the third concept on the 4th step. Similar regularity is typical for the subsequent steps.

Describing the results in general, the following should be noted:

- all models operate correctly regarding the influence transfer: the direction of changing concept states correspond to the signs of influences;

- all models are stable: pulse decays with time, which results in transferring the system in a stable state; 
- according to the results of modeling the state of each concept has changed in the same direction for all models (the states of the first and second concepts have increased, the state of the third one has decreased in comparison with the initial one), these results are generally consistent with intuitive understanding of the nature of system changes, which also proves the correctness of models;

- differences in predictions obtained by means of different models are quite well explained by the assumptions (concerning the nature of influences between concepts), which they are based on.

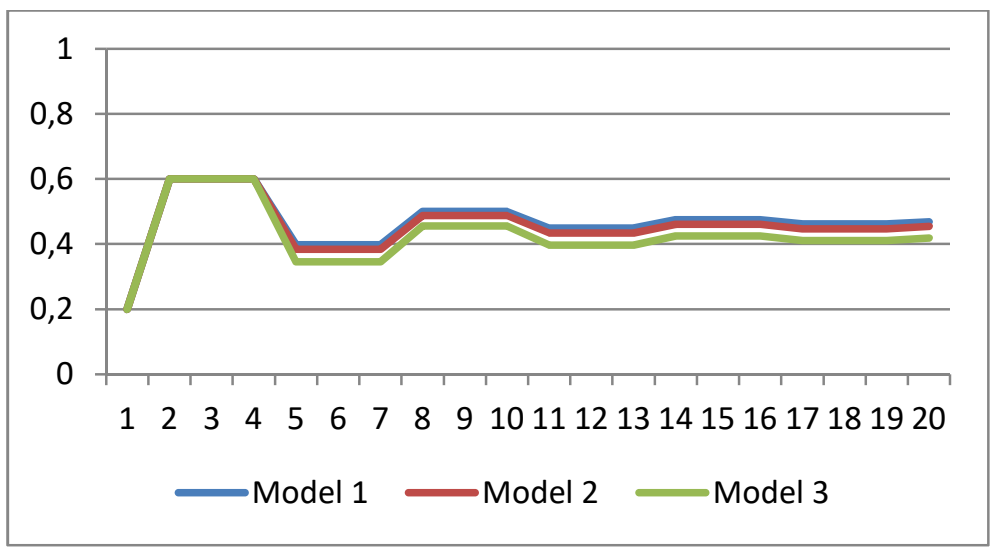

Fig. 2. Dynamics of state change of concept 1.

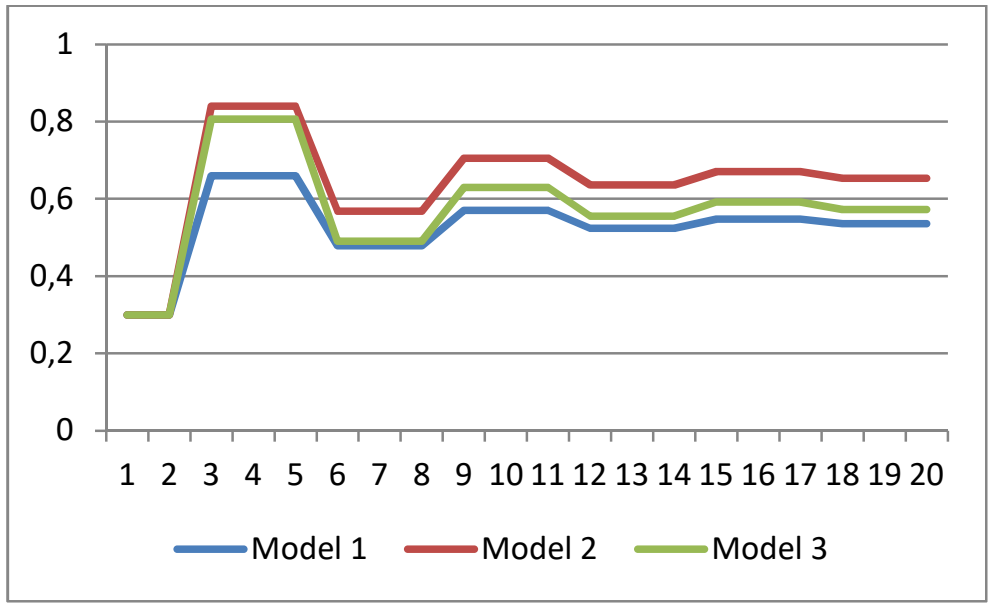

Fig. 3. Dynamics of state change of concept 2.

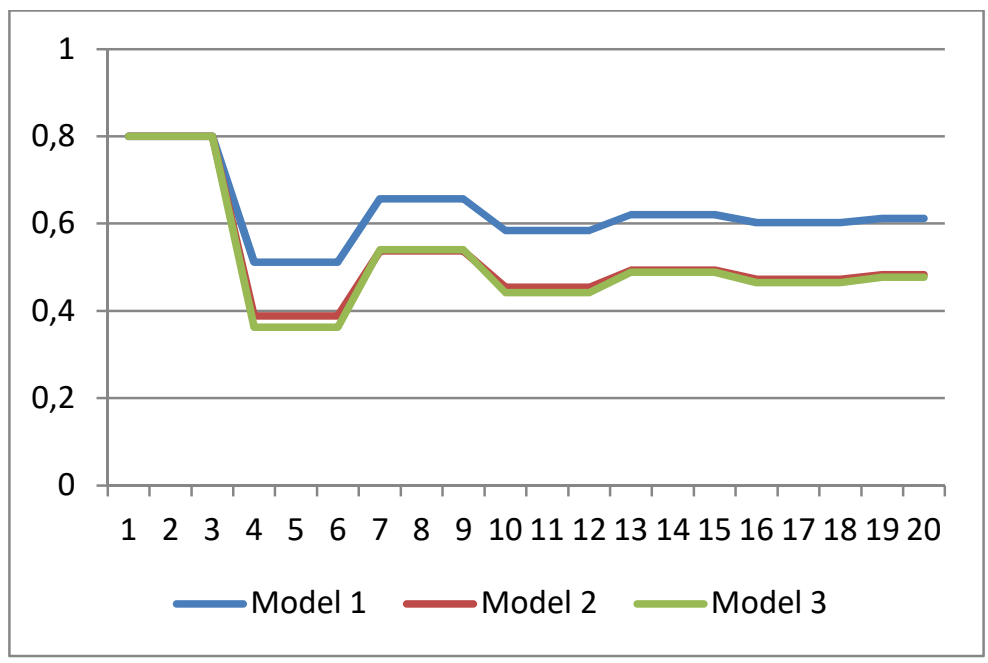

Fig. 4. Dynamics of state change of concept 3.

\section{Conclusion}

The paper considers a generalized model of pulse process for Sylov's fuzzy cognitive maps. This model, on the one hand, represents a generalization of previously developed models, and on the other hand, can serve as a basis for building other variations of pulse process. 
Also, there are proposed alternative implementations of this generalized model of pulse process, involving different interpretations of concept interactions. Experimental validation of these implementations is carried out, and its results confirm their correctness and operability.

Among the possible directions for further research, the following are of the greatest interest:

- identifying characteristics and making requirements to the methods of expert identification of FCM parameters in different models of pulse process;

- identifying characteristics and making requirements to the methods of identification of FCM parameters on the basis of statistical data in different models of pulse process;

- development of methods for selecting an optimal model of pulse process on the basis of the analysis of available statistical and expert data.

\section{References}

[1] Avdeeva ZK, Kovriga SV, Makarenko DI. Cognitive modeling for solving semi-structured management systems (situations). Managing Large Systems 2007; 16: 26-39. (in Russian)

[2] Averchenkov VI, Kozhukhar VM, Podvesovskii AG, Sazonova AS. Monitoring and Prediction of Regional Demand for Highest Scientific Degree Specialists: monograph. Bryansk: Bryansk State Technical University Press, 2010; 163 p. (in Russian)

[3] Borisov VV, Kruglov VV, Fedulov AS. Fuzzy Models and Networks. Moscow: "Goryachaya Liniya - Telekom” Publisher, 2012; 284 p. (in Russian)

[4] Erokhin DV, Lagerev DG, Laricheva EA, Podvesovskii AG. Strategic Enterprise Innovation Managemnet: monograph. Bryansk: Bryansk State Technical University Press, 2010; 196 p. (in Russian)

[5] Podvesovskii AG, Lagerev DG, Korostelyov DA. Application of fuzzy cognitive models for construction of alternatives set in decision problems. Bulletin of Bryansk State Technical University, 2009; 4(24): 77-84. (in Russian)

[6] Roberts FS. Discrete Mathematical Models with Application to Social, Biological and Environmental Problems. Prentice-Hall, Englewood Cliffs, 1976.

[7] Sylov VB. Strategic Decision Making in Fuzzy Environment. Moscow: "INPRO-RES" Publisher, 1995; 228 p. (in Russian) 